\title{
The Zero Model by Using Coincidence Scintillation (ZoMBieS) Method of Absolute Radioactivity Measurement
}

\author{
Lindsey J. Bignell, Li Mo, Tristan Steele, and S. Reza Hashemi-Nezhad
}

\begin{abstract}
A new method for the absolute radioactivity measurement of radionuclides using a liquid scintillation detector comprising three photomultipliers has been described. The method does not contain any a priori model of the photomultiplier detection efficiency and uses a modified Compton Coincidence technique, therefore it has been named the Zero Model By using Coincidence Scintillation (ZoMBieS) method. Validation measurements of the activity of a ${ }^{63} \mathrm{Ni}$ source against the Triple to Double Coincidence Ratio method are presented and the results agree within their standard uncertainties.
\end{abstract}

Index Terms-Liquid scintillators, scintillator characterization, standardization.

\section{INTRODUCTION}

A BSOLUTE radioactivity measurements using a Liquid Scintillation (LS) detector are generally achieved via the CIEMAT/NIST [1] or Triple to Double Coincidence Ratio (TDCR) [2] methods. The Compton Source Efficiency Tracing (CSET) method has also recently been developed [3] and appears to be a promising [4] alternative. These techniques provide accurate results for a range of nuclides and are particularly well suited for the measurement of nuclides with low energy emissions owing to the excellent detection efficiency obtainable with LS detectors.

The CIEMAT/NIST, TDCR and CSET methods model the single-Photomultiplier Tube (PMT) efficency of the $i$ th PMT for an incident energy deposit $E$ using an assumption that the process obeys Poisson statistics

$$
\epsilon_{i}(E)=1-e^{-\lambda_{i} E Q(E)}
$$

where $\lambda_{i}$ is the mean number of photoelectrons generated at the PMT photo-cathode per unit energy deposit in the scintillator and is treated as a constant. It is assumed in (1) that the PMT

Manuscript received October 10, 2012; revised May 01, 2013; accepted July 28, 2013. Date of publication September 04, 2013; date of current version October 09,2013

L. J. Bignell is with the Activity Standards Laboratory, Australian Nuclear Science and Technology Organisation, Lucas Heights, NSW 2234 and the School of Physics, University of Sydney, NSW 2006 Australia (e-mail: lindsey.bignell@ansto.gov.au; bignell@physics.usyd.edu.au).

L. Mo and S. R. Hashemi-Nezhad are with the School of Physics, University of Sydney, NSW 2006 Australia (e-mail: limo@physics.usyd.edu.au; reza@physics.usyd.edu.au).

T. Steele is with the Electronics Unit, Research School of Physics and Engineering, The Australian National University, ACT 0200 (e-mail: tristan.steele@ieee.org).

Color versions of one or more of the figures in this paper are available online at http://ieeexplore.ieee.org.

Digital Object Identifier 10.1109/TNS.2013.2275990 discrimination level has been set below the single photoelectron level but above the pedestal noise. $Q(E)$ is a correction for the non-linearity of the scintillator light yield, modeled using the Birks equation [5]

$$
E Q(E)=\int_{0}^{E} \frac{d E}{1+k B \frac{d E}{d x}}
$$

where $k B$ is an experimentally determined parameter. Although results calculated using the Birks equation are generally in agreement with other methods of absolute radioactivity determination, the detector model and choice of $k B$ is often the largest source of uncertainty in the measurement, particularly for radionuclides which decay with low energy emissions.

In order to investigate whether the ultimate measurement uncertainty can be reduced and also to provide a deeper insight into the non-linear energy response of scintillation detectors, a new absolute radioactivity measurement technique has been devised. This scintillation-based technique need not rely upon any model of the PMT detection efficiency, though it does assume that its three photodetectors are statistically independent. The detection efficiency is measured directly, using a modified Compton coincidence technique [6]. Since the new method uses the TDCR method and Compton coincidence and it does not depend on any model, this technique has been named the Zero Model By using Coincidence Scintillation (ZoMBieS) method. The ZoMBieS method can be applied to any 3 photomultiplier scintillation detection system and enables the direct measurement of the detection efficiency as a function of energy - opening the possibility for its use as another scintillator characterization technique useful for researchers studying scintillator non-linearity.

\section{THEORY}

Consider the ratio of the triple coincidence count rate $(T)$ to double coincidence count rate between PM tubes $\mathrm{X}$ and $\mathrm{Y}(X Y)$ for a monoenergetic energy deposit in the sensitive detection volume of a scintillation detector with three independent channels. This ratio will converge to the ratio of the triple coincident detection efficiency $\left(\epsilon_{T}\right)$ to the coincident detection efficiency between tubes $\mathrm{X}$ and $\mathrm{Y}\left(\epsilon_{X Y}\right)$ over a sufficiently long counting time. Furthermore, the ratio of $\epsilon_{T}$ to $\epsilon_{X Y}$ may be written as

$$
\frac{\epsilon_{T}}{\epsilon_{X Y}}=\frac{\epsilon_{X} \epsilon_{Y} \epsilon_{Z}}{\epsilon_{X} \epsilon_{Y}}=\epsilon_{Z}
$$

where $\epsilon_{i}$ is the detection efficiency of the $i$ th photomultiplier. The $T / X Y$ ratio for a monoenergetic energy deposit in the scintillation detector converges to the detection efficiency of the third channel. By using the Compton coincidences between the 


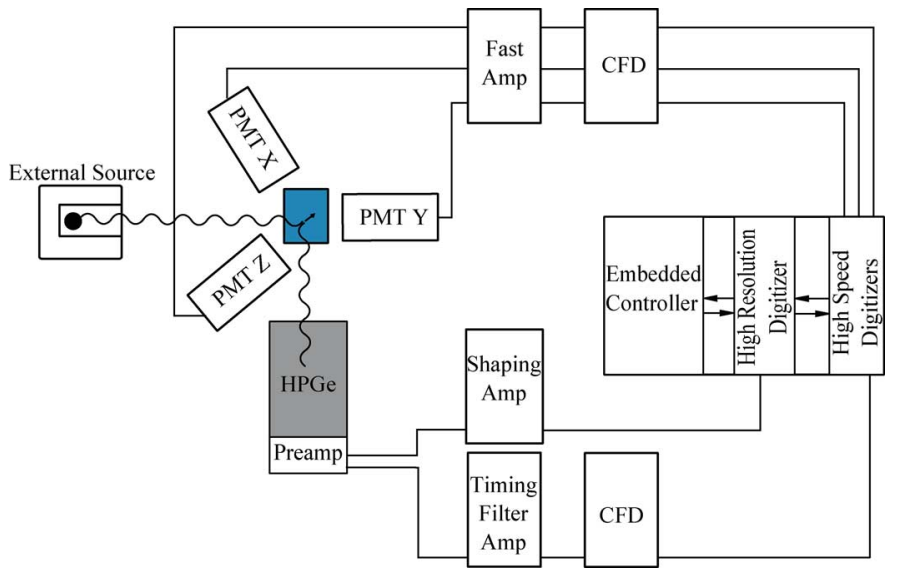

Fig. 1. Experimental setup used for the measurement. The rectangular region between the photomultipliers represents the liquid scintillator.

scintillator and HPGe detector, one may infer the energy deposit for every coincident interaction. Thus, a spectrum of efficiency may be generated spanning the range of available Compton electron energies. The breadth of this spectrum depends upon the $\gamma$-ray energy, the solid angle presented to the source by the scintillator and the solid angle presented to the HPGe detector by the source.

Consider now a measurement of a liquid scintillation detector bearing a radionuclide $R$ which deposits a spectrum $S(E)$ in the detector from $E=0$ up to some maximum $E_{m} \mathrm{keV}$. If a spectrum of efficiency can be generated which matches the energy range of $S(E)$, then the triple coincidence detection efficiency to $R\left(\epsilon_{X Y Z}(R)\right)$ may be determined by

$$
\epsilon_{X Y Z}(R)=\int_{0}^{E_{m}} S(E) \epsilon_{X}(E) \epsilon_{Y}(E) \epsilon_{Z}(E) d E
$$

whence the radioactivity may readily be determined from the count rate. If the efficiency spectra generated using scintillatorHPGe coincidence gating were measured in identical experimental conditions to the TDCR measurement, then these data may be used in (4).

\section{EXPERIMENTAL APPARATUS}

A diagrammatic representation of the detection system is shown in Fig. 1. The liquid scintillation detector housing was fabricated using $6 \mathrm{~mm}$ thick plastic (HDPE) walls to reduce unwanted scatter in the Compton coincidence measurements. The inner surfaces were painted with white $\mathrm{TiO}_{2}$-based paint to maximize the light collection. Three Hamamatsu R331-05 photomultipliers were used for detection.

A collimated ${ }^{51} \mathrm{Cr}$ source was used as the source of gamma rays. The spectrum of gamma rays that interact with the scintillator was investigated using a Monte Carlo simulation written in Geant4 version 9.5 to ensure that excessive scatter events did not affect the measurement. The simulation reproduced the geometry of the experimental detector used for the ZoMBieS measurements. The Lawerence Livermore National Laboratory low energy electromagnetic physics models were used [7]. To

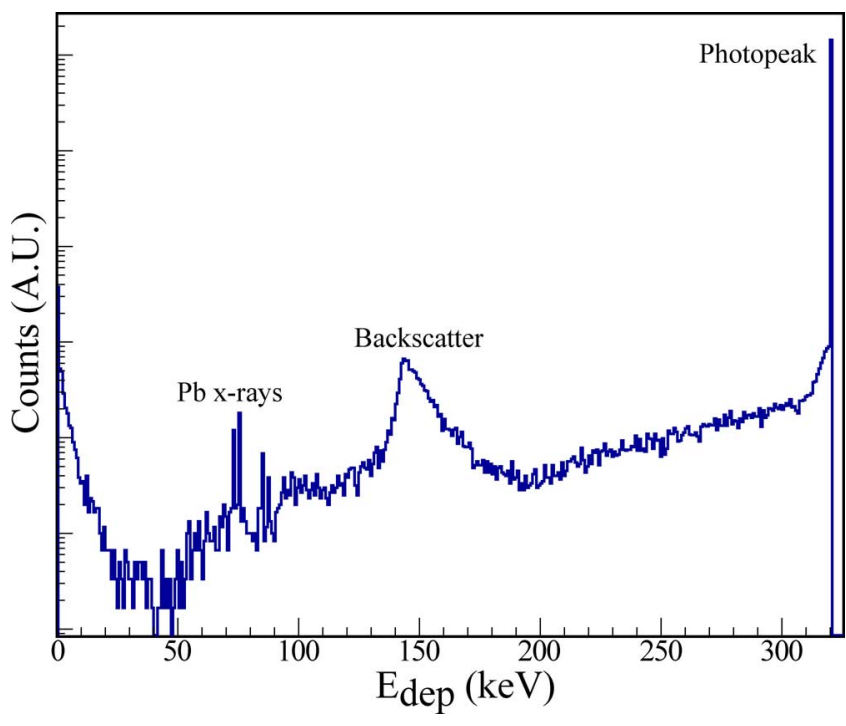

Fig. 2. Simulated spectrum of $\gamma$-rays which interact with the scintillator due to a collimated ${ }^{51} \mathrm{Cr}$ source.

decrease the simulation time, the $320 \mathrm{keV} \gamma$-rays emitted by the collimated source were given an initial momentum direction sampled randomly at $\pm 5^{\circ}$ about the axis of the lead collimator, which was sufficient to sample the entire opening of the collimator. The simulated spectrum (Fig. 2) indicates that the source can be treated as a monoenergetic source at the time it interacts with the scintillator $-97 \%$ of the spectrum falls within the $0.47 \mathrm{keV}$ wide photopeak bin. The peak at $150 \mathrm{keV}$ is due to Compton backscattering in the detector geometry prior to interaction with the scintillator.

${ }^{51} \mathrm{Cr}$ was chosen as a the external $\gamma$-ray source as it provided the best balance between two unwanted effects of excessive attenuation in the surrounding materials, which can be a problem at lower energies, and insufficient interaction with the scintillator, which can be a problem at higher energies. ${ }^{51} \mathrm{Cr}$ also fulfilled the criteria of having a monoenergetic emission and a sufficiently long half life for practical measurements.

The signals from the liquid scintillation and HPGe detectors were fed into National Instruments digitizers housed in a PXI chassis. The HPGe detector energy signal was acquired using a 12 bit, 200 MSPS NI PXI-5124 digitizer and the three PMT signals were acquired using two 8 bit, 1 GSPS NI PXI-5153 digitizers. Triggering was achieved using a HPGe fast timing channel with a suitably set CFD threshold, input into one of the PXI-5153 digitizers. The acquisition was controlled by an embedded controller running LabView. The HPGe energy spectrum was determined by least squares fitting of the acquired waveform to a Gaussian and the CFD inputs were analyzed using threshold timing.

The timing spectrum associated with the coincidences between the three PMT channels is presented in Fig. 3. The data in the figure are normalized to the total number of acquired counts in the spectrum, so that the ordinate corresponds to the probability of a given delay. A coincidence resolving time of $40 \mathrm{~ns}$ was used to evaluate triple and double coincidence events to enable comparison with the TDCR measurements, which were 


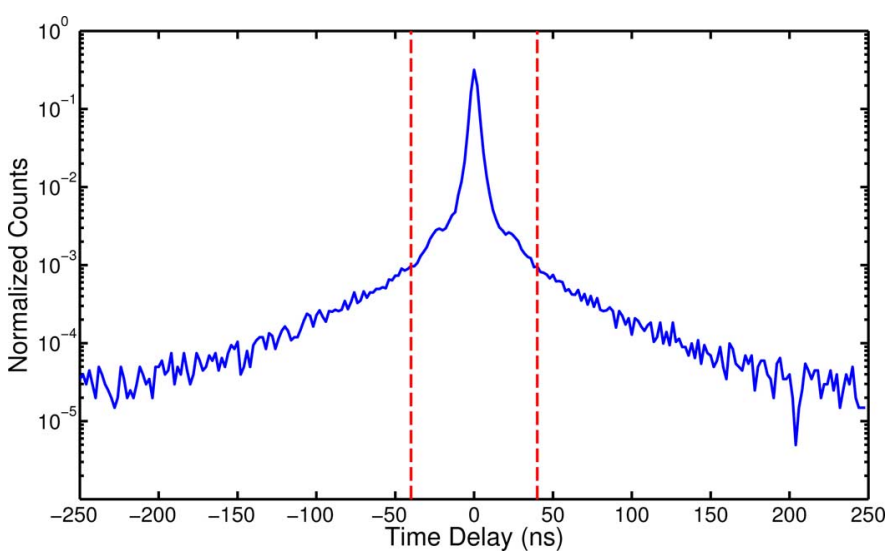

Fig. 3. Delay time histogram between the PMT channels, averaged over all channels. The PMT coincidence resolving time is indicated by the vertical dashed lines.

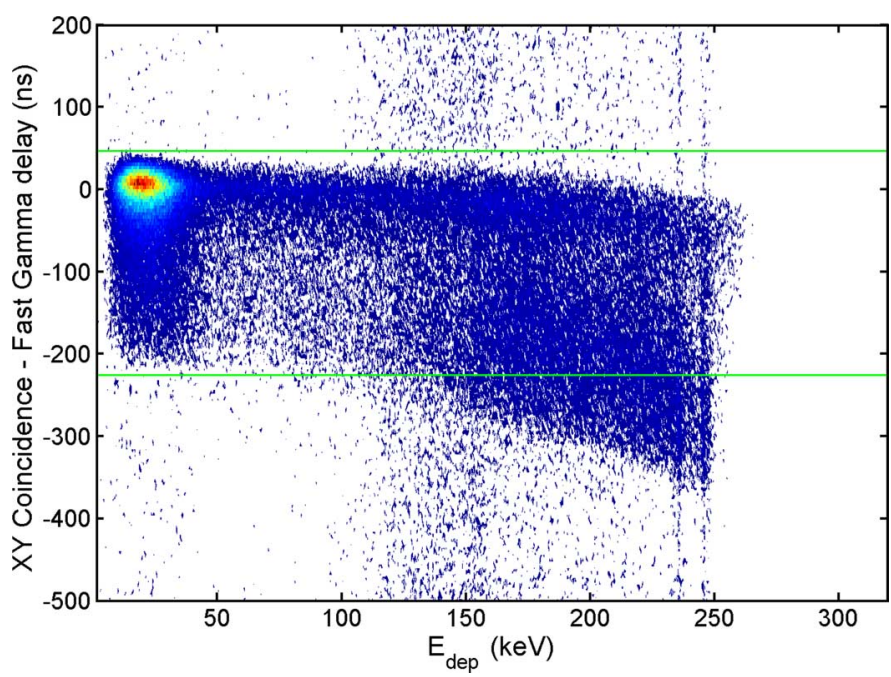

Fig. 4. 2D intensity plot of the inferred energy deposit in the scintillator and the time delay between the XY coincidences and the HPGe fast trigger. On the vertical axis, 0 refers to the trigger time. The horizontal lines are the upper and lower limits used in the background subtraction algorithm (see text for details).

acquired using the MAC3 coincidence module [8] with a coincidence resolving time of $40 \mathrm{~ns}$.

Fig. 4 presents an example of the number of coincident events occurring between two of the PMTs as a function of both the energy deposit in the scintillator and the registered mean arrival time of the PMT pulses. Minimal timing walk of approximately $28 \mathrm{~ns}$ over a $2.5 \mathrm{~V}$ pulse height difference was observed due to the use of a CFD on the HPGe fast trigger channel. The ZoMBieS efficiency spectra were generated by taking the ratio of the double coincidences to the triple coincidences after subtracting the background from these data. The background subtraction algorithm relied upon the definition of an upper and lower limit of the mean arrival time of the PMT pulses. Any counts registered above the upper limit or below the lower limit were considered to be background. Between the limits, the energy-dependent background was taken to be the mean of the average number of counts per bin above the upper limit and below the lower limit.

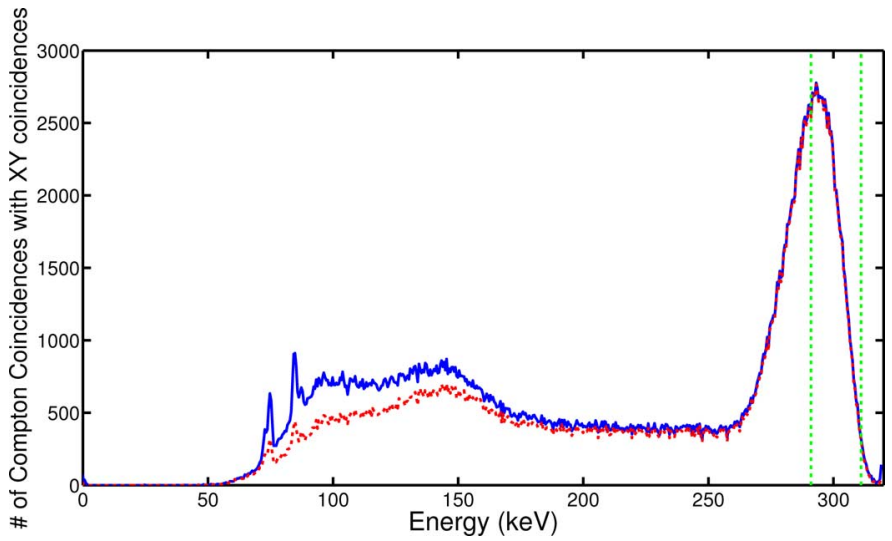

Fig. 5. Example spectrum of XY Compton coincident events. The solid trace used the entire dataset presented in Fig. 4 and the dashed trace used only the portion of the dataset between the upper and lower background subtraction limits of Fig. 4. The vertical dashed lines indicate the portion of these data kept for fitting the efficiency spectrum.

The spectrum of energy deposits in the scintillator are presented in Fig. 5. These data are the projection of the data in Fig. 4 onto the horizontal axis. The main peak in the spectrum is due to true coincidence events between the scintillator and the HPGe detector, and the lower energy continuum is due to accidental coincidences and events which interact with the scintillator before losing energy through scattering in the surrounding material and then being detected by the HPGe detector. The accidental coincidence component appears to be smaller than the scatter component, as can be seen by the strong time correlation in the low energy events with the coincident TDCR pulse arrival time. In order to mitigate any systematic error associated with the scatter events, only a narrow range of the coincidence data were used for generating the efficiency spectra. The range used for the current example is indicated in Fig. 5.

The timing distribution between the HPGe fast trigger channel and the XY coincident TDCR pulse arrival time is presented in Fig. 6. These data are the projection of the data in Fig. 4 on to the vertical axis. The observed background count rate was quite low, particularly around the energy region used for determining the efficiency. The tailing observed in the distribution appears to be correlated to some extent with the lower energy scattered events as illustrated by the differences between the two traces in Fig. 6.

\section{VALIDATION MEASUREMENTS}

${ }^{63} \mathrm{Ni}$ was chosen as the radionuclide for the ZoMBieS method validation measurements. It decays by allowed $\beta^{-}$decay to the daughter ground state, producing a spectrum of $\beta^{-}$particles with an endpoint energy of $66.98 \mathrm{keV}$. The relatively low energy $\beta$ particle spectrum makes measurement challenging. The low energy emissions also provide a rigorous test of the technique. This is because much of the spectrum probes a region where the scintillation detection efficiency is not near unity, so that the measurement is more sensitive to any errors in the ZoMBieS efficiency spectra.

The ${ }^{63} \mathrm{Ni}$ source was dispensed using a plastic pycnometer into $10 \mathrm{~mL}$ of Insta-Gel liquid scintillator in a glass vial. The 


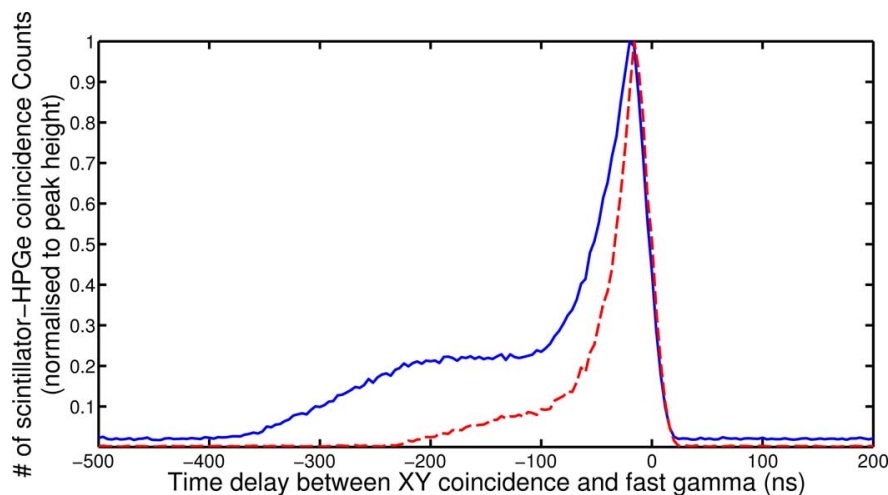

Fig. 6. Example of the timing distribution obtained between the HPGe fast trigger and the XY coincidences. The solid trace used the entire dataset presented in Fig. 4 and the dashed trace used only the portion of the same dataset whose energies fell between the vertical dashed lines in Fig. 5.

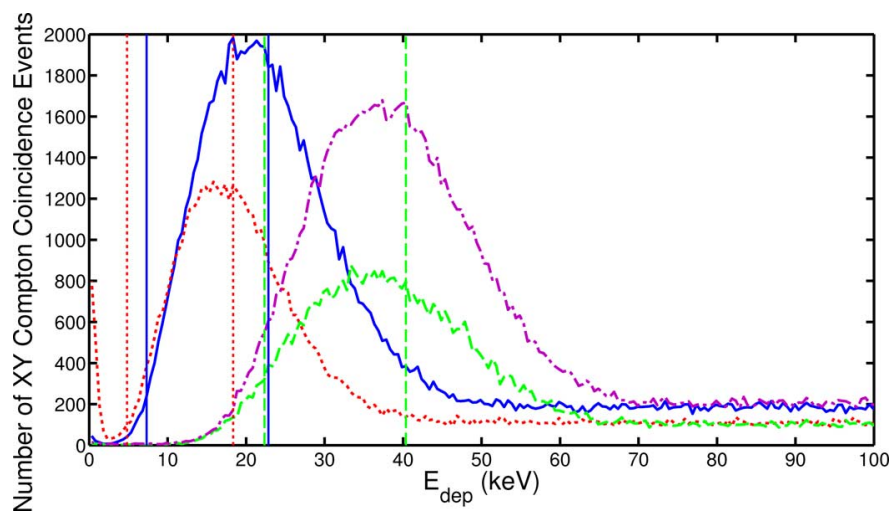

Fig. 7. Spectra of XY-HPGe coincident events used in the activity measurement of Source A. The dotted, solid, dashed, and dash-dotted traces represent scattering angles of $23^{\circ}, 27^{\circ}, 36.5^{\circ}$, and $36.5^{\circ}$, respectively. The vertical lines of identical line-type indicate the limits between which the data were used to determine the efficiency spectrum. The dashed and dash-dotted traces share the same limits.

source was agitated and allowed to settle prior to measurement. This source will hereafter be referred to as Source A.

Conventional TDCR measurements were performed on Source A using a custom TDCR detector that has been described previously [9], [10]. The data were analyzed using the TDCR11 program [11] to determine the detection efficiency.

For the ZoMBieS measurements, four large data sets were acquired at various angles to probe different parts of the efficiency spectrum. Spectra of the collected Compton coincidences with the XY-coincident TDCR events are presented in Fig. 7. A small excess of accidental coincidence events involving the ${ }^{51} \mathrm{Cr}$ photopeak were observed in one of the acquisitions. This was because the collimated $\gamma$-ray beam was very nearly directly aligned with the HPGe collimation in this measurement in order to sample low Compton scatter angles. The lower limit cut on the data was chosen to discriminate these events.

Figs. $8-10$ present the efficiency spectra associated with PMTs X, Y, and Z. Least squares regressions were performed against two fit functions, of the form

$$
f(x)=A e^{-B x}+C
$$

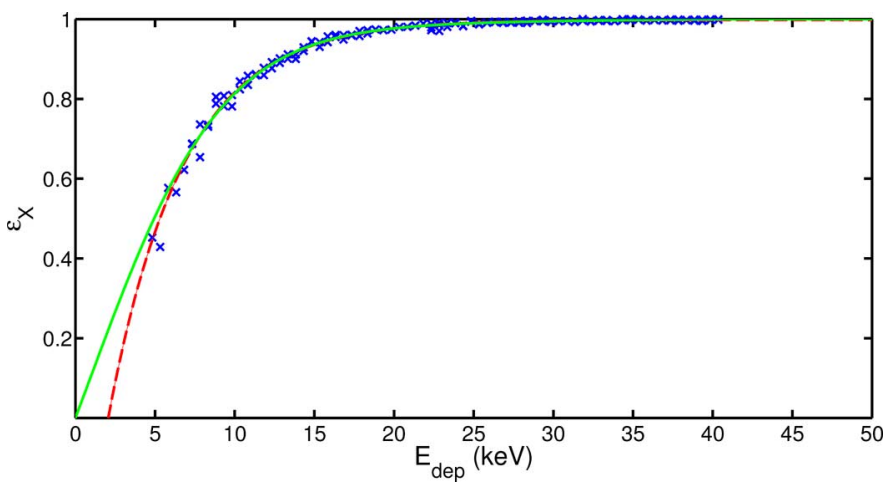

Fig. 8. Efficiency of PMT X (Fig. 1) as a function of energy deposit in Source A. The dashed trace represents the fit of the data to the Arbitrary model (5) and the solid trace represents the fit of the data to the NLP model (6).

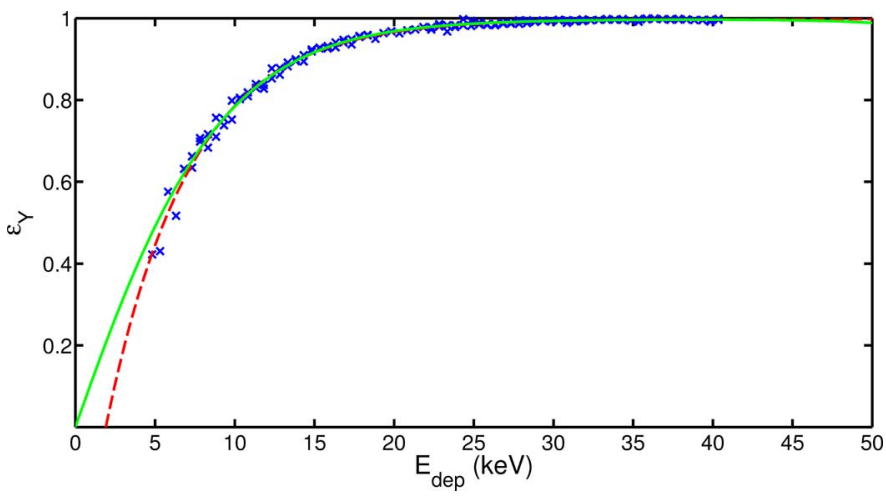

Fig. 9. Efficiency of PMT Y (Fig. 1) as a function of energy deposit in Source A. The dashed trace represents the fit of the data to the Arbitrary model (5) and the solid trace represents the fit of the data to the NLP model (6).

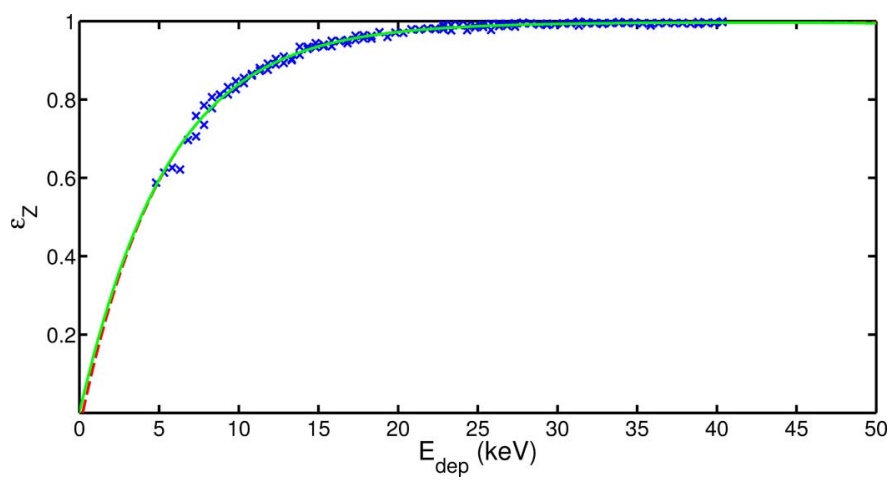

Fig. 10. Efficiency of PMT Z (Fig. 1) as a function of energy deposit in Source A. The dashed trace represents the fit of the data to the Arbitrary model (5) and the solid trace represents the fit of the data to the NLP model (6).

and

$$
g(x)=1-e^{-\left(c_{1} x+c_{2} x^{2}+c_{3} x^{3}\right)} .
$$

$A, B, C, c_{1}, c_{2}$, and $c_{3}$ are fit parameters. The form of (5) was chosen solely from considerations of the observed shape of the efficiency spectrum and without any consideration of the physical validity of the fit function. The form of (6) was chosen so that it followed the form of (1), with the PMT non-linearity approximated using a cubic polynomial. Equation (6) therefore models the PMT response as a Poisson statistical process in 
TABLE I

Fit PARAMETERS FOR FITS IN Figs. $8-10$.

THe PARAMETERS ARE DeFined IN (5) AND (6)

\begin{tabular}{|c|c|c|c|}
\hline Parameter & PMT X & PMT Y & PMT Z \\
\hline \multicolumn{4}{|c|}{ Arbitrary Model } \\
\hline$A$ & -1.53 & -1.43 & -1.04 \\
\hline$B$ & 0.214 & 0.191 & 0.189 \\
\hline$C$ & 0.998 & 0.998 & 0.997 \\
\hline \multicolumn{4}{|c|}{ NLP Model } \\
\hline$c_{1}$ & 0.1143 & 0.1105 & 0.1755 \\
\hline$c_{2}$ & $6.91 \times 10^{-3}$ & $5.45 \times 10^{-3}$ & $1.28 \times 10^{-3}$ \\
\hline$c_{3}$ & $-1.5 \times 10^{-4}$ & $1.2 \times 10^{-4}$ & $5.4 \times 10^{-5}$ \\
\hline
\end{tabular}

keeping with the currently used measurement techniques, but does not assume any model of the scintillator non-linearity. The fitting models introduced in (5) and (6) will hereafter be referred to as the Arbitrary and Non-Linear Poisson (NLP) models, respectively. The fit parameters obtained by least squares regression are given in Table I.

Given the unphysical nature of the Arbitrary model fit at low energy deposits (negative efficiency), a linear extrapolation to the origin was performed below $5 \mathrm{keV}$. Although it would be preferable to have sufficient data at low energies to achieve a better model fit, the systematic error introduced by this extrapolation was relatively small. The ${ }^{63} \mathrm{Ni}$ triple coincidence detection efficiency, $\epsilon_{X Y Z}\left({ }^{63} \mathrm{Ni}\right)$ was calculated using (4). From these calculations, energy range covering the extrapolated portion of the Arbitrary model efficiency fit contributes only $\sim 1 \%$ to $\epsilon_{X Y Z}\left({ }^{63} \mathrm{Ni}\right)$. The effect upon the calculated detection efficiency caused by simply following the model prediction and not using any extrapolation to zero was to decrease $\epsilon_{X Y Z}\left({ }^{63} \mathrm{Ni}\right)$ by $0.14 \%$.

The NLP model fits to the efficiency spectra tended to diverge from unit efficiency at higher energy deposits, due to a lack of data at these energies to constrain the fit. Therefore, the detection efficiency was set to 1 above $40 \mathrm{keV}$ to avoid any systematic effects.

A striking feature of the Arbitrary model fits is how closely the fit of PMT Z resembles the behavior of a perfectly linear detector that obeys Poisson statistics, for which $A=-1, C=$ 1 , and $B$ is a constant. This result was not expected as liquid scintillators are known to exhibit non-linearity in this energy range due to ionization quenching, as is observed in PMT X and $Y$. The ability of the ZoMBieS method to directly measure the scintillator response is useful for validating the existing model of PMT detection efficiency and will be the subject of future studies.

The activity concentration of Source A was calculated using (4). This was compared to that calculated using conventional TDCR with $k B=0.011 \mathrm{~cm} \mathrm{MeV}^{-1}$. The triple coincidence detection efficiency was used for the ZoMBieS method calculation. The results are presented in Table II. No statistically significant deviation was observed between the two ZoMBieS method activities and the TDCR Method activity.
TABLE II

MEASured Source A ACTIVITy Using the TDCR AND ZoMBieS Method [DETERMINEd Using the Two FITTING Models Described By (5) AND (6)] Relative to the Mean of the Three Results. STANDARD UNCERTAINTIES ARE GIVEN

\begin{tabular}{ccc}
\hline \hline Method & Activity $(\mathrm{kBq} / \mathrm{g})$ & Activity relative to mean \\
\hline TDCR & $651.4 \pm 2.8$ & $0.9945 \pm 0.0043$ \\
ZoMBieS, Arbitrary Model & $658.0 \pm 4.5$ & $1.0044 \pm 0.0070$ \\
ZoMBieS, NLP Model & $655.8 \pm 4.7$ & $1.0011 \pm 0.0072$ \\
\hline \hline
\end{tabular}

TABLE III

UnCERTAINTY BUdGet FOR THE MEASUREMENT OF THE RADIOACTIVITY Concentration of Source A Using the TDCR Method. Relative STANDARD UNCERTAINTIES ARE GIVEN

\begin{tabular}{cc}
\hline \hline Uncertainty component & TDCR Method \\
\hline$k B$ & $15 \%$ \\
TDCR & $0.16 \%$ \\
${ }^{63} \mathrm{Ni}$ specrum endpoint & $0.02 \%$ \\
Detector Instability & $0.14 \%$ \\
Statistical count rate uncertainty & $0.09 \%$ \\
Source Mass & $0.11 \%$ \\
\hline Combined Uncertainty & $0.43 \%$ \\
\hline \hline
\end{tabular}

TABLE IV

UNCERTAINTY BUDGET FOR THE ZOMBIES METHOD MEASUREMENT OF Source A Using the Arbitrary Model AND the NLP Model of DETECTION EFFICIENCY. RELATIVE STANDARD UNCERTAINTIES ARE GIVEN

\begin{tabular}{ccc}
\hline \hline Uncertainty component & Arbitrary Model & NLP Model \\
\hline Fitting uncertainty & $0.64 \%$ & $0.64 \%$ \\
Extrapolation to origin & $0.07 \%$ & N/A \\
Detector Instability & $0.15 \%$ & $0.15 \%$ \\
Count Rate & $0.10 \%$ & $0.10 \%$ \\
Source Mass & $0.11 \%$ & $0.11 \%$ \\
\hline Combined Uncertainty & $0.68 \%$ & $0.72 \%$ \\
\hline
\end{tabular}

\section{UNCERTAINTY ANALYSIS}

The uncertainty budgets detailing the combined measurement uncertainty of the TDCR and ZoMBieS method activity measurements are presented in Tables III and IV. The uncertainty analysis was carried out in a manner consistent with the ISO Guide for the Expression of Uncertainty in Measurement [12].

\section{A. Components Common to Both Techniques}

The following uncertainty components were considered in the determination of the combined measurement uncertainty for both techniques.

1) The Measured Coincidence Count Rate: Triple coincidences were used in the determination of the ZoMBieS activity as these had the lowest relative uncertainty in the efficiency of the measured coincidences and because triple coincidences make full use of the information available from each of the PMTs. The TDCR measurements used the logical sum of double coincidence count rate in the determination of the activity. Both techniques used the statistical standard deviation as the type A standard uncertainty associated with the coincident count rate. 


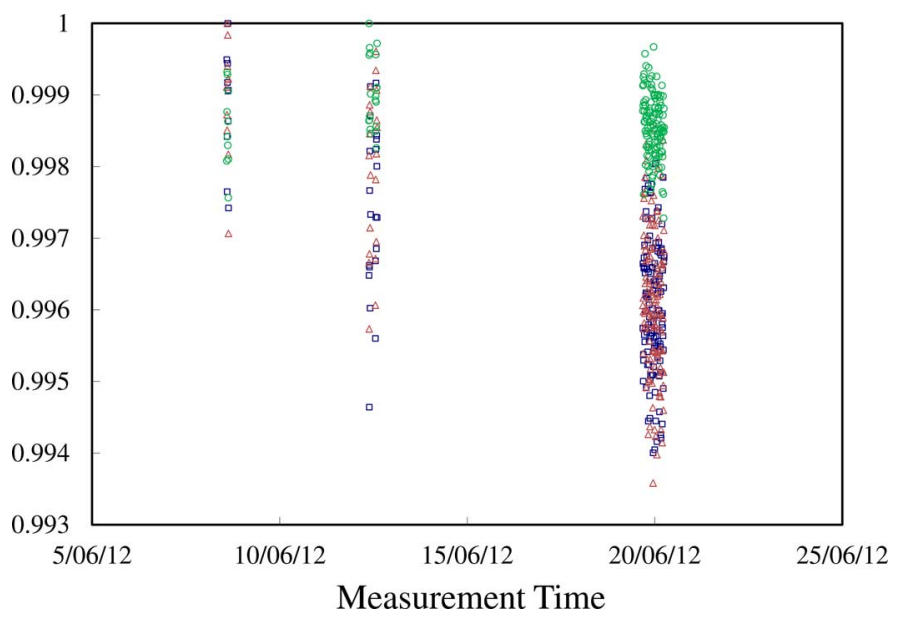

Fig. 11. Measured count rates in the logical sum of double coincidence (squares) and triple coincidence (triangles) channels. The triple to double coincidence ratio is also indicated (circles). All data are normalized to the maximum acquired value.

The sensitivity coefficient relating the uncertainty of the coincidence count rate to the uncertainty in the activity measurement was calculated analytically.

2) The Scintillation Detector Stability: Repeated measurements of the same measurement geometry some days apart were taken to check the stability of the detector without moving the source between measurements or shutting down the PMT bias supply. A small amount of instability was observed in the triple and double count data, probably due to PMT degradation (Fig. 11). The standard deviation of the mean was taken as the type A standard uncertainty associated with the instability in the detector. The detector stability and the statistical uncertainty associated with the evaluation of the coincidence count rate were taken as two equally weighted independent uncertainty components associated with the activity measurement. The sensitivity coefficient relating the uncertainty of the detector stability to the uncertainty in the activity measurement was calculated analytically.

3) Source Mass: Both sources were dispensed using plastic pycnometers and weighing was conducted with a microbalance calibrated with a set of weights traceable to the Australian National Standard. The type B standard uncertainty in the mass determination was estimated to be $0.01 \mathrm{mg}$. The sensitivity coefficient relating the uncertainty of the dispensed mass to the uncertainty in the activity measurement was calculated analytically.

\section{B. TDCR Measurements}

The following uncertainty components were considered in the determination of the conventional TDCR combined measurement uncertainty.

1) The Determination of $k B$ : The $k B$ value was determined by choosing values which gave the smallest slopes on the activity vs TDCR plots of Fig. 12. The uncertainty in $k B$ was evaluated by examining how stable this slope minimum was to the removal of data from the plot. The removal of a single TDCR

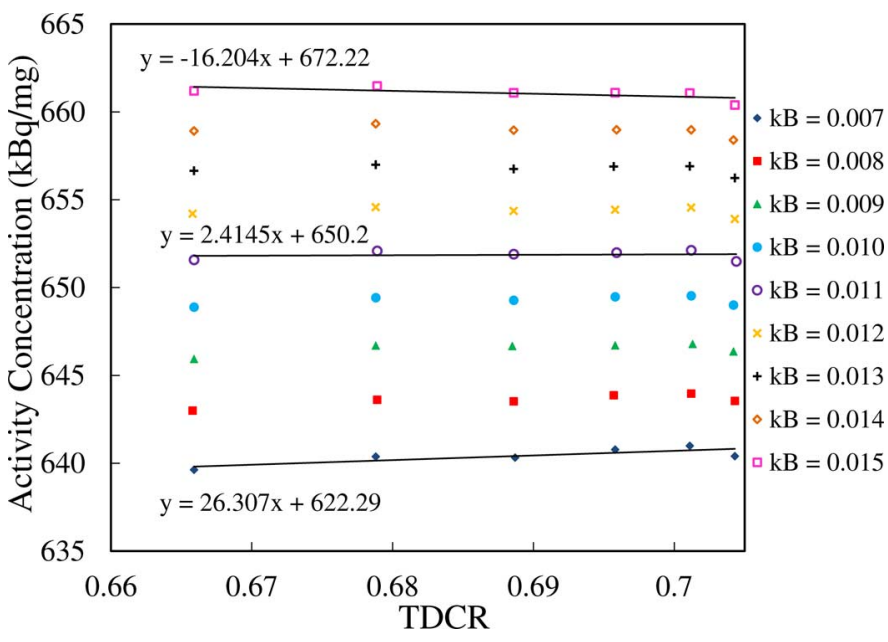

Fig. 12. Measurement of the Birks parameter, $k B$, for Source A. $k B$ was determined to be $0.011 \pm 0.0015 \mathrm{~cm} \mathrm{MBq}^{-1}$.

measurement from Fig. 12 led to the minimum slope being associated with $k B=0.008 \mathrm{~cm} \mathrm{MeV}^{-1}$. This was considered to be an extreme case and the difference of $0.003 \mathrm{~cm} \mathrm{MeV}^{-1}$ was taken to be the expanded type $\mathrm{B}$ uncertainty, $k=2$. The $k B$ uncertainty was taken as one of the independent components of the uncertainty in the logical sum of double detection efficiency $\left(\epsilon_{D}\right)$. The sensitivity coefficient associated with $k B$ used in the determination of the efficiency uncertainty was calculated numerically for both sources. The sensitivity coefficient relating the uncertainty of $\epsilon_{D}$ to the uncertainty in the activity measurement was calculated analytically.

2) The Triple to Double Coincidence Ratios: The three TDCR uncertainties were considered to be entirely due to statistical fluctuations in the count rates. As these values are very strongly correlated, the largest individual TDCR uncertainty was taken as representative of the entire TDCR uncertainty component. The TDCR type A uncertainty was taken as one of the independent components of the uncertainty in $\epsilon_{D}$. The sensitivity coefficient associated with the TDCR used in the determination of the efficiency uncertainty was calculated numerically for both sources. The sensitivity coefficient relating the uncertainty of $\epsilon_{D}$ to the uncertainty in the activity measurement was calculated analytically.

3) The ${ }^{63} \mathrm{Ni} \beta$ Spectrum Endpoint Energy: The value and uncertainty in the $\beta$ spectrum endpoint energy was taken from the Decay Data Evaluation Project table of recommended values [13]. Unfortunately no values or uncertainties associated with the $\beta$ spectrum shape could be found for ${ }^{63} \mathrm{Ni}$, and the default shape factor for allowed transitions equal to 1 was used and this uncertainty component neglected. The half life of ${ }^{63} \mathrm{Ni}$ is quite long and this uncertainty component was taken to be negligible. The spectrum endpoint type B uncertainty was taken as one of the independent components of the uncertainty in $\epsilon_{D}$. The sensitivity coefficient associated with the $\beta$ spectrum endpoint energy used in the determination of the efficiency uncertainty was calculated numerically for both sources. The sensitivity coefficient relating the uncertainty of $\epsilon_{D}$ to the uncertainty in the activity measurement was calculated analytically. 


\section{Zombies Measurements}

The following uncertainty components were considered in the determination of the ZoMBieS method combined measurement uncertainty.

1) Fitting the Efficiency Spectrum: The efficiency spectra were fit using a linear least squares regression against the fit functions defined in (5) and (6). The fit uncertainty was determined using the prediction bounds returned by the fitting algorithm to calculate the detection efficiency, and taking these calculated detection efficiencies as the extrema associated with the same confidence interval as the prediction bounds.

The sensitivity coefficient relating the uncertainty of $\epsilon_{T}$ to the uncertainty in the activity measurement was calculated analytically.

2) Extrapolation to the Origin in the Arbitrary Model: The fitting algorithm for the Arbitrary model was not required to pass through the origin, and the obtained fits were considered too physically unrealistic to use due to the presence of negative efficiencies. A simple linear extrapolation to the origin from the fit value at the lowest energy datum in the coincidence data was used. The uncertainty in this extrapolation was estimated by considering the triple coincidence efficiency calculated without an extrapolation as the expanded uncertainty $(k=2)$ extremum. The extrapolation type B uncertainty was taken with the fitting uncertainty as one of the two independent and equally weighted components of the uncertainty of $\epsilon_{T}$ for the ZoMBieS measurements. The sensitivity coefficient relating the uncertainty of $\epsilon_{T}$ to the uncertainty in the activity measurement was calculated analytically.

\section{DISCUSSION}

The statistical agreement of the ZoMBieS method activities with those calculated using the TDCR method is a strong validation of the technique. The combined measurement uncertainties associated with the ZoMBieS method measurements were comparable to, though larger than, the combined measurement uncertainty associated with the TDCR Method measurement.

These validation measurements have been performed using ${ }^{63} \mathrm{Ni}$. It is expected that the ZoMBieS method can easily be adapted to other pure $\beta$ emitting sources with a higher energy $\beta$ spectrum or electron capture radionuclides in a similar manner to existing liquid scintillation based methods. Based upon the results obtained, the fitted efficiency model can safely be assumed to be 1 above $\sim 40 \mathrm{keV}$ for our detector, so that extrapolating beyond this point should not cause any significant systematic uncertainty.

The main limitation of the ZoMBieS method is the very long measurement time that is required to obtain results. The Compton coincidence process is very inefficient - typical scintillator-HPGe coincidence count rates were $0.5-0.75$ counts per second. The results presented in this work required approximately 1.5 months of measurement time. Aside from the practical limitations associated with long measurements this limitation also raises the issue of photomultiplier stability. TDCR measurements are generally conducted using short acquisition times during the day and therefore measurements are not subjected the day-night temperature variations experienced during using the ZoMBieS method. Furthermore, after such a significant measurement time, it is likely that the scintillator will start to degrade [14], leading to a systematic uncertainty. It is therefore imperative that the measurements times be improved, without compromising the quantity of data collected.

Simple ways to improve the count rate include increasing the external source activity, increasing the efficiency of either of the HPGe or scintillation detectors to the incoming $\gamma$-rays, and bringing the external source, scintillation detector and HPGe detector closer together without compromising the collimation. Increasing the volume of the liquid scintillator to boost the interaction probability may be of benefit provided any reductions in scintillation detection efficiency are minimal.

Temperature controlled cooling may also be advantageous. The PMT dark noise decreases quite substantially at low temperatures and the the scintillation efficiency increases at low temperatures. Temperature control would also help to improve the stability of the detector.

Measurement of a greater number of scintillator-HPGe coincidence events would enable a better characterization of the scintillation efficiency spectrum, thereby enabling fits with lower statistical uncertainty. Measurement over a shorter time period would minimize any systematic effects associated with scintillator degradation. Better control of the measurement conditions may also reduce scatter about the fit caused by detector instability during the long measurements. As the fitting uncertainty was the major contributer to the total measurement uncertainty of the ZoMBieS method measurements, reducing the uncertainty of this component should be considered a priority. It is possible that with some additional detector design optimization, this technique could achieve combined measurement uncertainties smaller than those that are obtained with the TDCR method.

Another challenging aspect of the ZoMBieS technique is the limit of available energies. The ${ }^{51} \mathrm{Cr} 320 \mathrm{keV} \gamma$-ray was chosen as the external excitation source in order to provide a balance between the competing effects of low interaction probability with the scintillator at higher energies and excessive attenuation and scattering by the structural materials at lower energies. However it would be of interest both in terms of performing better fits to the efficiency spectra and in terms of understanding the scintillation process at low energy deposits if lower energy data could be acquired.

\section{CONCLUSION AND FUTURE WORK}

We have validated the ZoMBieS method against the TDCR method using a ${ }^{63} \mathrm{Ni}$ source in a liquid scintillation detector. The measurements agreed within their standard uncertainties, with the ZoMBieS method uncertainties being slightly larger than the TDCR uncertainties. It is possible that with further optimization of the experimental technique, the ZoMBieS uncertainties could be further reduced.

Although the ZoMBieS method need not depend upon any particular model of the detection efficiency, the measured liquid scintillation detection efficiency spectra are described well by the Poisson model with a non-linear response. As the statistical distribution associated with photon detection is likely to be Poisson-like for any scintillator type, this opens the possibility of studying other scintillator matrices. 
While the primary motivation for the development of the ZoMBieS method is absolute radioactivity measurement, the method may also be used to study the response of scintillators at very low light levels down to a single photon per PMT. As a scintillator characterization tool, the ZoMBieS method may measure the scintillator non-linearity of organic liquid and plastic scintillators, as well as any other scintillator that gives less than 100\% PMT detection efficiency. The use of ZoMBieS for this purpose will be explored in future work.

\section{ACKNOWLEDGMENT}

The authors would like to thank A. Stopic from the Nuclear Activation Analysis group at ANSTO for the production of the ${ }^{51} \mathrm{Cr}$ source.

\section{REFERENCES}

[1] A. G. Malonda and E. G. Torano, "Evaluation of counting efficiency in liquid scintillation counting of pure $\beta$-ray emitters," Int. J. Appl. Radiat. Isotopes, vol. 33, no. 4, pp. 249-253, 1982.

[2] K. Pochwalski, R. Broda, and T. Radoszewski, "Standardization of pure beta emitters by liquid-scintillation counting," Int. J. Appl. Radiat. Isotopes A, vol. 39, no. 2, pp. 165-172, 1988.

[3] P. Cassette and P. Do, "The Compton source efficiency tracing method in liquid scintillation counting: A new standardisation method using a TDCR counter with a Compton Spectrometer," Appl. Radiat. Isotopes, vol. 66, pp. 1026-1032, 2008.
[4] P. Cassette, F. Jaubert, and I. Tartes, "Study of the influence of the liquid scintillator in the Compton efficiency tracing method," Appl. Radiat. Isotopes, vol. 68, pp. 1510-1514, 2010.

[5] J. B. Birks, "Scintillations from organic crystals: Specific fluorescence and relative response to different radiations," in Proc. Phys. Soc., 1951, vol. 64, pp. 874-877.

[6] J. D. Valentine and B. D. Rooney, "Design of a Compton spectrometer experiment for studying scintillator non-linearity and intrinsic energy resolution," Nucl. Instrum. Meth. A, vol. 353, no. 1-3, pp. 37-40, 1994.

[7] J. Apostolakis, S. Giani, M. Maire, P. Nieminen, M. G. Pia, and L. Urban, "Geant4 Low Energy Electromagnetic Models for Electrons and Photons," Tech. Rep., CERN, 1999, CERN-OPEN-99-034.

[8] J. Bouchard and P. Cassette, "MAC3: An electronic module for the processing of pulses delivered by a three photomultiplier liquid scintillation counting system," Appl. Radiat. Isotopes, vol. 52, no. 3, pp. $669-672,2000$.

[9] M. J. Qin, L. Mo, D. Alexiev, and P. Cassette, "Construction and implementation of a TDCR system at ANSTO," Appl. Radiat. Isotopes, vol. 66, no. 6-7, pp. 1033-1037, 2008.

[10] T. Steele, L. Mo, L. Bignell, M. Smith, and D. Alexiev, "FASEA: A FPGA acquisition system and software event analysis for liquid scintillation counting," Nucl. Instrum. Meth. A, vol. 609, no. 2-3, pp. 217-220, 2009.

[11] P. Cassette, Private Communication 2002.

[12] "Guide to the Expression of Uncertainty in Measurement," Tech. Rep., International Organisation for Standardisation, 1993.

[13] M.-M Bé, V. Christé, C. Dulieu, E. Browne, C. Baglin, V. Chechev, N. Kuzmenco, R. Helmer, F. Kondev, D. MacMahon, and K. B. Lee, "Monographie BIPM-5, Table of Radionuclides," Tech. Rep., Bureau International de Poids et Mesures, 2006.

[14] L. Mo, L. J. Bignell, T. Steele, and D. Alexiev, "Activity measurements of ${ }^{3} \mathrm{H}$ using the TDCR method and observation of source stability," Appl. Radiat. Isotopes, vol. 68, no. 7-8, pp. 1540-1542, 2010. 